\title{
Prolonged Risk of Suicide Reattempts in Patients with Alcohol Use Disorder and Acute Alcohol Use: A Register-Based Follow-Up Study (2010-2015)
}

\author{
Jinhee Lee', Sei Jin Chang'2, Hyun Kim³, Min-Hyuk Kim', \\ Joung-Sook Ahn', Ki-Chang Park ${ }^{1}$, Jin Sil Moon ${ }^{4}$, and Seongho Min ${ }^{1 凶}$ \\ ${ }^{1}$ Department of Psychiatry, Yonsei University Wonju College of Medicine, Wonju, Republic of Korea \\ ${ }^{2}$ Department of Preventive Medicine, Yonsei University Wonju College of Medicine, Wonju, Republic of Korea \\ ${ }^{3}$ Department of Emergency Medicine, Yonsei University Wonju College of Medicine, Wonju, Republic of Korea \\ ${ }^{4}$ Center of Biomedical Data Science (CBDS), Yonsei University Wonju College of Medicine, Wonju, Republic of Korea
}

\begin{abstract}
Objective This study aimed to clarify the role of acute alcohol use and alcohol use disorder in individuals exhibiting suicidal behaviors, including repetition of suicide attempt.

Methods Data pertaining to 691 suicide attempters who had visited an emergency center and had been followed-up from 2010 to 2015 were gathered. Participants were categorized into following three groups according to alcohol use pattern at the time of the suicide attempt: 1) suicide attempters with neither alcohol use disorder nor acute alcohol consumption (NAU), 2) suicide attempters who had used alcohol during the suicide attempt but did not have alcohol use disorder (AAU), and 3) suicide attempters with alcohol use disorder (AUD). Group comparisons and multivariate Cox proportional models for suicidal behavior were used for statistical analysis.

Results AUD have been shown to have lower lethality of suicide attempt but higher risk of suicide reattempts in the future. Furthermore, positive relationships between suicide reattempts and AUD persisted throughout the longer period than other groups.

Conclusion Suicide attempters with AUD should be considered a high-risk group for suicide reattempts in future, and this group should be followed-up for a longer period with specialized care program.

Psychiatry Investig 2019;16(2):145-153
\end{abstract}

Key Words Addictive disorder, Suicide, Mood disorder.

\section{INTRODUCTION}

Alcohol is one of the leading causes of the global burden of disease, and alcohol use disorders (AUDs) are known to be a major risk factor for suicide. ${ }^{1}$ AUDs are the second most common psychiatric disorder among those exhibiting suicidal behaviors worldwide, following major depression. ${ }^{2}$ Further, about $40 \%$ of patients with AUD have had at least one suicide attempt at some point. ${ }^{3}$ A large cohort study conducted in Denmark indicated that suicide mortality was higher in

Received: July 29, 2018 Revised: November 15, 2018

Accepted: November 22, 2018

$\triangle$ Correspondence: Seongho Min, MD, PhD

Department of Psychiatry, Yonsei University Wonju College of Medicine, 20 Ilsan-ro, Wonju 26426, Republic of Korea

Tel: +82-33-741-1260, Fax: +82-33-741-0532, E-mail: mchorock@yonsei.kr

(c) This is an Open Access article distributed under the terms of the Creative Commons Attribution Non-Commercial License (https://creativecommons.org/licenses/bync/4.0) which permits unrestricted non-commercial use, distribution, and reproduction in any medium, provided the original work is properly cited. patients with AUD than it was in any other psychiatric illness (unadjusted hazard ratio: 7.98). ${ }^{4}$ On the other hand, acute alcohol use(AAU) increases the risk of suicidal behavior, which indicates that alcohol consumption at the time of suicide is not merely a reflection of ongoing drinking problems. ${ }^{5}$ Further, about $37 \%$ (10-69\%) of suicide decedents and 40\% (10-73\%) of suicide attempters were found to have used alcohol at the time of the suicide attempt. ${ }^{2}$ Several studies have examined the overlapping effect of AUD and AAU on suicide attempts. ${ }^{6}$ One previous study suggested that individuals attempting suicide under the influence of alcohol were more likely to have AUD $(30-49 \%)^{7}$ and other studies found that the rate of AAU was higher in individuals with AUD in completed suicides $(69 \% \text { vs. } 23 \%)^{8}$ and in suicide attempts $(83 \%$ vs. $31 \%){ }^{9}$ However, since the alcohol use patterns are often duplicated and it is difficult to distinguish clearly, there are few studies that separately examine the effects of the AUD and AAU on suicidal behavior. 
The suicide rate in Korea is the second highest among the Organization for Economic Co-Operation and Development (OECD) member countries, with 25.8 deaths per 100,000 people, which is approximately 2.2 times higher than the OECD average of 11.6 deaths per 100,000 people..$^{10}$ Further, compared to western countries and other Asian countries, the suicide rates in Korea presented a sharper increase and stronger persistence. In Korea, which has such a high suicide rate, the per capita consumption of alcohol is $7.95 \mathrm{~L}$, and the annual drinking rate of adults is close to $77 \%$, which is far above the world average according to the WHO statistics published in 2014. This unusual alcohol use rate might allow to suggest the presence of a strong association with the high suicide rates in Korea. Although controversy still exists with the alcohol use pattern in relation to the risk factors for suicide reattempts, alcohol use has been known to be associated with repeated suicide attempts. ${ }^{11}$ However, studies on the association between alcohol use pattern and suicide reattempts are lacking in Korea, and very little is known about the effect of alcohol use in prolonged risk of suicide reattempt.

In these context, we aimed to outline the transient and prolonged effects of alcohol use patterns on suicide attempters. The main objectives of the present study were: 1 ) to assess the characteristics of suicidal behaviors according to alcohol use patterns; 2) to compare the lethality and intent of suicide attempters by alcohol use patterns; 3 ) to investigate the prolonged risks of repeated suicide attempts by alcohol use patterns. To our knowledge, this is the first study to compare the characteristics of suicidal behaviors within groups according to non-overlapping patterns of alcohol use, using a Korean sample who have both high rates of suicide and alcohol consumption at the same time.

\section{METHODS}

\section{Study sample}

Study subjects were 1659 suicide attempters who visited Gangwon Western Region Emergency Medical Center during the 6-year period from March 1, 2010, to December 31, 2015. A suicide attempt was defined as self-destructive behavior with the intention of ending one's life, leading to a patient's admission to the emergency room. ${ }^{12}$ Data on 691 suicide attempters were finally analyzed, excluding those who were aged less than 18 years, were not available to evaluate alcohol use history, and had refused the case management program intervention (Figure 1). All subjects were fully informed about the aims and methods of the study and the case management program, and informed consent was obtained prior to participation.

\section{Baseline measures and assessment instruments}

\section{Sociodemographic characteristics}

After physical assessment by an emergency department physician, a psychiatric interview was conducted with all patients, including sociodemographic variables such as age, gender, education level, marital status, religion, employment, monthly income, medical illness, and smoking status.

\section{Characteristics of presenting suicide behaviors}

The characteristics of suicide behaviors presented were assessed for all patients, including current suicide ideation (absent/present/uncheckable), suicide plan (absent/present), last will (absent/present), prior suicide attempts (absent/present), method of suicide attempt (poisoning/gas inspiration/hanging/sword/fall down/others), help seeking (no/provide a clue/ seeking help before the attempt/seeking help after the attempt), and medical lethality (low; no damage or mild physical damage/high; severe physical damage requiring intensive treatment), and the following scales were administered.

\section{Risk-Rescue Rating Scale}

The Risk-Rescue Rating Scale (RRRS) was used to assess the perceived severity of the current suicide attempt. The RRRS measures suicide risk based on five suicide risk factors and five rescue factors. The RRRS score is calculated by dividing the risk coefficient with the sum of the risk coefficient and rescue coefficient, with lower scores indicating lower lethality of the suicide attempt. Higher RRRS scores suggest severe lethality with a high possibility of completing suicide. ${ }^{13}$

\section{Suicidal Intent Scale}

The Suicidal Intent Scale (SIS) was developed to assess the risk of suicide and severity of suicidal intent. It is used widely because it quantifies suicidal intentions and has been validated, exhibiting a high correlation with suicide risk. ${ }^{14}$ The SIS score was calculated by summing the circumstances score (eight items) and self-report score (seven items), with higher scores indicating higher suicide intention.

\section{Categorization of alcohol use patterns}

To evaluate the alcohol drinking pattern of suicide attempters, we assessed the consumption of alcohol at the time of the suicide attempt and used a three-items subscale of the Alcohol Use Disorders Identification Test (AUDIT-C) ${ }^{15}$ Acute alcohol use was considered present if the suicide attempters consumed alcohol before and/or at the time of the suicide attempt. Male suicide attempters who had AUDIT-C scores of 7 or higher, and females with scores of 6 or higher were considered to have alcohol use disorder. The higher cut-off scores of 


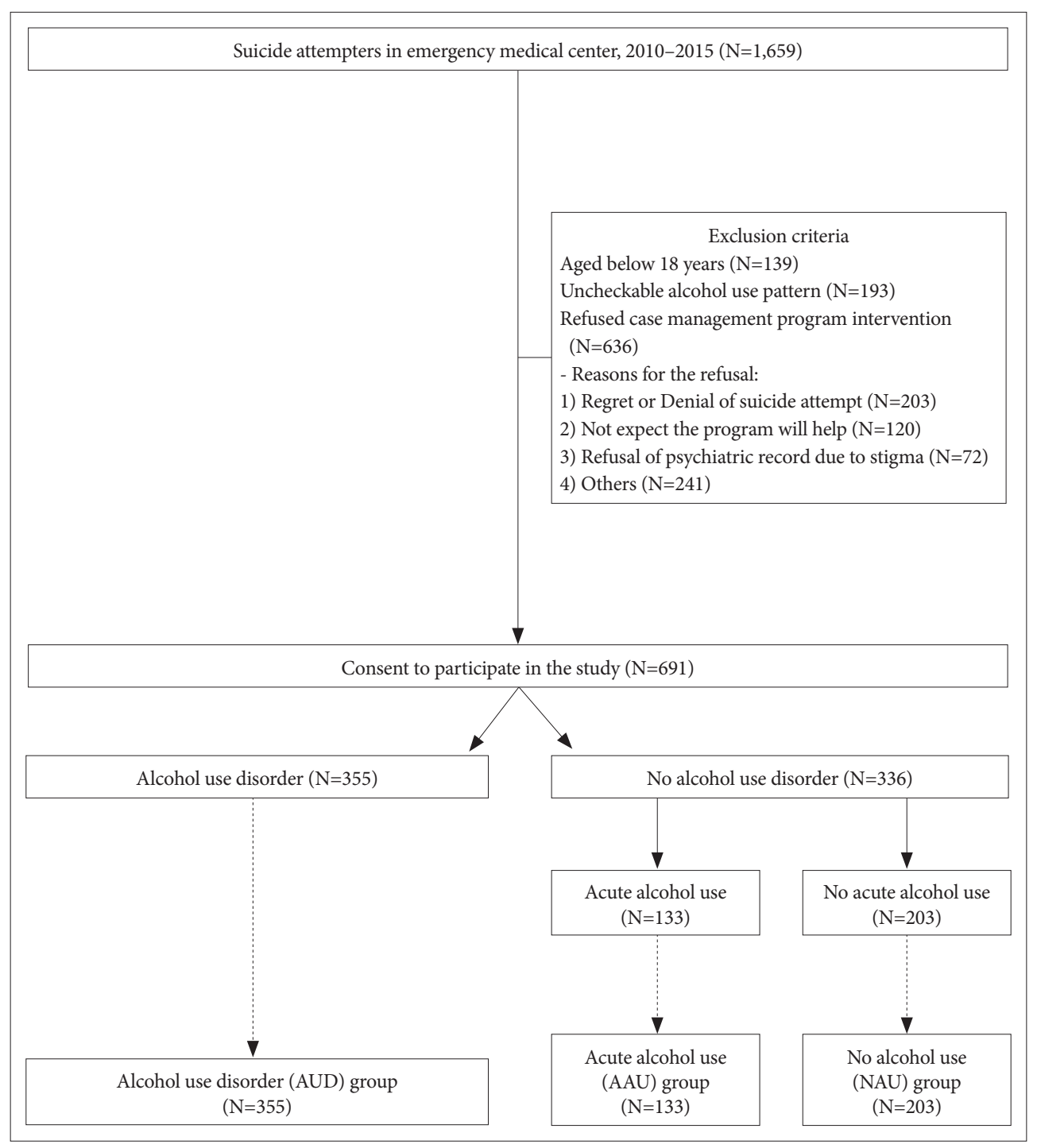

Figure 1. Study population.

the AUDIT-C in the Korean population as compared to that in other countries ( 4 or higher in men and 3 or higher in women $)^{16}$ represent the high frequency and quantity of alcohol drinking observed in Korea, and the validity of the cut-off scores has been confirmed in a previous study. ${ }^{17}$ Thus, based on the AUDIT-C scores, we divided the suicide attempters into the following three groups: 1) suicide attempters with neither alcohol use disorder nor acute alcohol consumption [NAU; $\mathrm{n}=203$ (29.4\%)], 2) suicide attempters who had used alcohol during the suicide attempt but did not have alcohol use disorder [AAU; $\mathrm{n}=133$ (19.2\%)], and 3) suicide attempters with alcohol use disorder [AUD; $\mathrm{n}=355$ (51.4\%)].

\section{Follow-up procedures}

Participants who consented to participation in the study have been followed up by trained investigators. The investigators were mainly psychiatric nurses and social workers, but the procedures and assessments of the study were guided and supervised by two psychiatrists, and weekly consensus meetings were held with the entire investigators, two psychiatry residents, and two psychiatrists. Each participant is assessed for repeat suicide attempts every 3 months, via face-to-face or over the phone interviews, and/or he/she is contacted at the time of suicide reattempt.

\section{Statistical analysis}

At baseline, we compared the sociodemographic and suicidal behavior characteristics of the three groups using a Pearson's chi square test and a one-way ANOVA. The RRRS and SIS scores were compared using the Scheffe's method. The primary endpoint was based on a survival analysis and was determined using the Cox proportional model. The length of 
follow-up for each subject was determined by the number of months between the baseline evaluation and the date of either a repeat suicide attempt or the end of the follow-up period. The results are reported as hazard ratios (HRs) with 95\% confidence intervals (CI) and p values from the Cox proportional hazard regression model. Furthermore, multivariate Cox proportional models were applied to examine group differences after controlling for demographic factors (age, sex, education level, marriage status, religion, employment, monthly income, smoking, and medical problems) and characteristics of suicide behaviors (suicide ideation, suicide plan, last will, past suicide attempt history, method of suicide attempt, medical lethality, and help seeking). Further, the Kaplan-Meier method was used to compare the point of suicide reattempt for the participants. All statistical analyses were performed using SPSS (version 23.0, IBM Corp., Armonk, NY, USA), and statistical significance was declared at $\mathrm{p}<0.05$.

\section{Ethics statement}

This study was approved by the Institutional Review Board of the Yonsei University Wonju College of Medicine (YWMR15-9-097). All the participants provided informed consent.

Table 1. Sociodemographic characteristics of subjects grouped by alcohol use pattern

\begin{tabular}{|c|c|c|c|c|c|}
\hline & (1) NAU, N (\%) & (2) AAU, N (\%) & (3) AUD, N (\%) & $\mathrm{t} / \mathrm{x}$ or $\mathrm{F}$ & p-value \\
\hline Total number & $203(29.4)$ & $133(19.2)$ & $355(51.4)$ & & \\
\hline Age, years $($ mean $\pm S D)(691)$ & $52.87 \pm 20.15$ & $47.11 \pm 16.54$ & $41.96 \pm 14.25$ & 28.00 & $\begin{array}{c}<0.001 \\
(3)>(2)>(1)\end{array}$ \\
\hline Gender (691) & & & & 36.00 & $<0.001$ \\
\hline Male & $62(30.5)$ & $42(31.6)$ & $190(53.5)$ & & \\
\hline Female & $141(69.5)$ & $91(68.4)$ & $165(46.5)$ & & \\
\hline Education level (674) & & & & 32.67 & $<0.001$ \\
\hline$\leq$ Elementary school & $83(41.3)$ & $30(23.1)$ & $68(19.8)$ & & \\
\hline Middle/high school & $90(44.8)$ & $79(60.8)$ & $226(65.9)$ & & \\
\hline$\geq$ College & $28(13.9)$ & $21(16.2)$ & $49(14.3)$ & & \\
\hline Marital status (683) & & & & 6.77 & 0.148 \\
\hline Single or never married & $45(22.3)$ & $24(18.0)$ & $98(28.2)$ & & \\
\hline Married/Cohabitation & $109(54.0)$ & $80(60.2)$ & $182(52.3)$ & & \\
\hline Separated/divorced/widowed & $48(23.8)$ & $29(21.8)$ & $68(19.5)$ & & \\
\hline Religion (675) & & & & 17.41 & $<0.001$ \\
\hline Absent & $101(50.5)$ & $74(56.5)$ & $234(68.0)$ & & \\
\hline Present & $99(49.5)$ & $57(43.5)$ & $110(32.0)$ & & \\
\hline Employment (691) & & & & 4.12 & 0.127 \\
\hline Unemployed & $75(36.9)$ & $35(26.3)$ & $117(33.0)$ & & \\
\hline Employed & $128(63.1)$ & $98(73.7)$ & $238(67.0)$ & & \\
\hline Monthly income(\$) (597) & & & & 15.61 & 0.016 \\
\hline$\leq 1000$ & $91(51.4)$ & $46(40.4)$ & $114(37.3)$ & & \\
\hline $1,000-2,000$ & $41(23.2)$ & $29(25.4)$ & $103(33.7)$ & & \\
\hline $2,000-3,000$ & $31(17.5)$ & $21(18.4)$ & $46(15.0)$ & & \\
\hline$\geq 3,000$ & $14(7.9)$ & $18(15.8)$ & $43(14.1)$ & & \\
\hline Medical problems (674) & & & & 27.92 & $<0.001$ \\
\hline No & $98(49.5)$ & $90(69.2)$ & $247(71.4)$ & & \\
\hline Yes & $100(50.5)$ & $40(30.8)$ & $99(28.6)$ & & \\
\hline Smoking (666) & & & & 95.70 & $<0.001$ \\
\hline Never smoker & $135(67.2)$ & $73(56.6)$ & $109(32.4)$ & & \\
\hline Ex-smoker & $19(9.5)$ & $18(14.0)$ & $17(4.1)$ & & \\
\hline Current smoker & $47(23.4)$ & $38(29.5)$ & $210(62.5)$ & & \\
\hline
\end{tabular}

Between-group comparisons were conducted using the t-test for continuous variables and the chi-square test for categorical variables. Due to missing data, N<691 for some variables. NAU: No Alcohol Use Group, AAU: Acute Alcohol Use Group, AUD: Alcohol Use Disorder Group 


\section{RESULTS}

\section{Sample characteristics}

The final sample included 691 suicide attempters, who were divided into the three groups according to the pattern of alcohol use in relation to the suicide attempt. The descriptive statistics and proportions of each alcohol use pattern among suicide attempters who visited the emergency room have been presented in Table 1. Among the suicide attempters, 19.2\% had AAU, and 51.4\% had AUD. And also 78.9\% of AUD group had drank alcohol at the time of suicide attempt. There were significant differences between the three groups in terms of age, gender, education level, religion, monthly income, medical problems, and smoking status but no differences were found for marital status and employment. The participants belonging to the NAU group were significantly older (52.87 \pm 20.15 vs. AAU: 47.11 \pm 16.54 and AUD: $41.96 \pm 14.25$; $<<0.001)$ and a larger proportion among them had medical problems $(50.5 \%$ vs. AAU: $30.8 \%$ and AUD: $28.6 \%$; $<<0.001$ ) as compared to the others. Further, the AUD group had the lowest proportion of those with a religion (30.9\% vs. NAU: $49.5 \%$ and AAU: $43.5 \%$; $\mathrm{p}<0.001)$ and medical problems ( $27.5 \%$ vs. NAU: $50.5 \%$ and AAU: $30.8 \%$; $<<0.001)$. Rate of current smoking was significantly higher in those with AUD as compared to the others (62.5\% vs. NAU: $23.4 \%$ and AAU: 29.5\%; p<0.001) (Table 1).

\section{Group comparison of suicidal behaviors}

The groups did not differ in terms of current suicide ideation, suicide plan, and last will. However, those with AUD showed a significantly higher rate of prior suicide attempts (37.4\% vs.

Table 2. Characteristics of presenting suicide behavior in the three groups

\begin{tabular}{|c|c|c|c|c|c|}
\hline & (1) NAU, N (\%) & (2) AAU, N (\%) & (3) AUD, N (\%) & $\chi^{2}$ & p-value \\
\hline Current suicide ideation (650) & & & & 7.898 & 0.095 \\
\hline Absent & $49(25.4)$ & $36(28.3)$ & $110(33.3)$ & & \\
\hline Present & $71(36.8)$ & $56(44.1)$ & $126(38.2)$ & & \\
\hline Uncheckable & $73(37.8)$ & $35(27.6)$ & $94(28.5)$ & & \\
\hline Suicide plan (658) & & & & 0.713 & 0.700 \\
\hline Absent & $158(83.6)$ & $109(85.8)$ & $295(86.3)$ & & \\
\hline Present & $31(16.4)$ & $18(14.2)$ & $47(13.7)$ & & \\
\hline Last will (671) & & & & 0.845 & 0.656 \\
\hline Absent & $178(90.8)$ & $116(88.5)$ & $314(91.3)$ & & \\
\hline Present & $18(9.2)$ & $15(11.5)$ & $30(8.7)$ & & \\
\hline Prior suicide attempts (674) & & & & 8.963 & 0.011 \\
\hline Absent & $147(74.2)$ & $94(71.8)$ & $216(62.6)$ & & \\
\hline Present & $51(25.8)$ & $37(28.2)$ & $129(37.4)$ & & \\
\hline Method of suicide attempt (691) & & & & 23.914 & 0.008 \\
\hline Poisoning & $161(79.3)$ & $97(72.9)$ & $250(70.4)$ & & \\
\hline Gas inspiration & $14(6.9)$ & $18(13.5)$ & $24(6.8)$ & & \\
\hline Hanging & $13(6.4)$ & $8(6.0)$ & $19(5.4)$ & & \\
\hline Sword & $10(4.9)$ & $8(6.0)$ & $52(14.6)$ & & \\
\hline Fall down & $2(1.0)$ & $0(0)$ & $3(0.8)$ & & \\
\hline Others & $3(1.5)$ & $2(1.8)$ & $7(2.0)$ & & \\
\hline Help seeking (661) & & & & 18.545 & 0.005 \\
\hline No & $136(69.4)$ & $76(58.9)$ & $175(52.1)$ & & \\
\hline Provide a clue & $18(9.2)$ & $24(18.6)$ & $62(18.5)$ & & \\
\hline Seeking help before the attempt & $3(1.5)$ & $4(3.1)$ & $12(3.6)$ & & \\
\hline Seeking help after the attempt & $39(19.9)$ & $25(19.4)$ & $87(25.9)$ & & \\
\hline Medical lethality (684) & & & & 8.121 & 0.017 \\
\hline Low & $170(84.2)$ & $119(89.5)$ & $321(92.0)$ & & \\
\hline High & $32(15.8)$ & $14(10.5)$ & $28(8.0)$ & & \\
\hline
\end{tabular}

Between-group comparisons were conducted using the t-test for continuous variables and the chi-square test for categorical variables. Due to missing data, $\mathrm{N}<691$ for some variables. NAU: No Alcohol Use Group, AAU: Acute Alcohol Use Group, AUD: Alcohol Use Disorder Group 
NAU: $25.8 \%$ and AAU: $28.2 \% ; \mathrm{p}=0.011$ ) as compared to the others (Table 2). They also tended to choose swords as a suicide method (14.6\% vs. NAU: $4.9 \%$ and AAU: $6.0 \%$; $=0.008$ ) than the others did. Help seeking, including providing a clue, and seeking help before and after an attempt were most common in the AUD group (47.9\% vs. NAU: $30.6 \%$ and AAU: 41.1\%; $\mathrm{p}=0.005$ ). The proportion of low medical lethality of suicide attempts is also higher in the AUD group than other groups ( $8.0 \%$ vs. NAU: $15.8 \%$ and AAU: $10.5 \%$; $\mathrm{p}=0.017$ ) (Table 2).

Further, those with AUD showed a lower RRRS score as compared to those in the NAU group (37.52 \pm 7.99 and $40.46 \pm$ 8.99 , respectively; $\mathrm{p}<0.001$ ), and a higher rescue score than those in both NAU and AAU groups $(12.46 \pm 2.05,11.64 \pm 2.17$, and $11.90 \pm 2.23$, respectively; $\mathrm{p}<0.001$ ), which means that those with AUD tended to attempt low-risk suicide with a high rescue opportunity. However, there was no significant difference between the AAU and the other groups in terms of the RRRS and SIS scores (Table 3).

\section{Effects of alcohol use on repetition of suicide attempts}

The mean follow-up period of participants was 13 months (SD 15.87) and ranged from 1 to 60 months. Table 4 shows that AUD was associated with increased HR of suicide reattempts before and after adjusting for covariates such as de- mographic and suicide-related variables; however, AAU was not associated with future suicide reattempts. Further, as compared to the NAU group, the AUD group was 2.55 times (95\% CI: 1.36-4.78) was more likely to repeat suicide attempts, before adjusting for other factors. Model 1 shows the HR after adjusting for sociodemographic factors (age, sex, education level, marriage status, religion, employment, monthly income, smoking, and medical problems), Model 2 shows the HR after adjusting for the characteristics of suicidal behavior (suicide ideation, suicide plan, last will, past suicide attempt history, method of suicide attempt, medical lethality, and help seeking), and Model 3 shows the HR after adjusting for both sociodemographic and suicide behavior characteristics. The HRs for the AUD group were higher for each model (HR: 2.96, 95\% CI: $1.23-7.10$ in Model 1; HR: 2.27, 95\% CI: 1.12-4.58 in Model 2; HR: 2.96, 95\% CI: 1.23-7.10 in Model 3) as compared to that for the NAU group. Further, as compared to the AAU group, the AUD group was not more likely to attempt suicide before adjusting covariates, but it was 2.43 times (95\% CI: 1.035.69) more likely to repeat suicide attempts after adjusting for demographic factors in Model 1.

Following an analysis of the reattempt period using the Kaplan-Meier method, it was found that 76 out of the 691 patients reattempted suicide; 12 (5.9\%) of the NAU group, $10(7.5 \%)$ of the AAU group, and 54 (15.2\%) of the AUD group $\left(\chi^{2}=13.44\right.$,

Table 3. Comparison of Risk-rescue Rating Scale and Suicidal Intent Scale scores in the three groups

\begin{tabular}{lrrrrc}
\hline & \multicolumn{1}{c}{$(1) \mathrm{NAU}$} & $(2) \mathrm{AAU}$ & $(3) \mathrm{AUD}$ & p-value & Group comparison \\
\hline RRRS (mean $\pm \mathrm{SD})$ & $40.46 \pm 8.99$ & $39.48 \pm 8.08$ & $37.52 \pm 7.99$ & $<0.001$ & $(1)>(3)$ \\
Risk (mean $\pm \mathrm{SD})$ & $7.97 \pm 2.31$ & $7.75 \pm 2.00$ & $7.51 \pm 1.99$ & 0.058 & - \\
Rescue $($ mean $\pm \mathrm{SD})$ & $11.64 \pm 2.17$ & $11.90 \pm 2.23$ & $12.46 \pm 2.05$ & $<0.001$ & $(1)<(3),(2)<(3)$ \\
SIS $($ mean $\pm \mathrm{SD})$ & $5.83 \pm 5.14$ & $6.18 \pm 5.55$ & $5.84 \pm 5.12$ & 0.807 & - \\
P1 (mean $\pm \mathrm{SD})$ & $3.21 \pm 2.64$ & $3.26 \pm 2.92$ & $3.14 \pm 2.63$ & 0.904 & - \\
P2 (mean $\pm \mathrm{SD})$ & $2.61 \pm 3.42$ & $2.93 \pm 3.49$ & $2.74 \pm 3.17$ & 0.720 & - \\
\hline
\end{tabular}

RRRS (Risk-Rescue Rating Scale) score=Risk score/(Risk score+Rescue score) $\times 100$, SIS (Suicidal Intent Scale) score=P1 (objective circumstances of the act) $+\mathrm{P} 2$ (subject's own perception of their feelings and thoughts at the time of the act). Comparisons were conducted using the ANCOVA, adjusted for age. The difference between groups was examined using the Scheffe's multiple comparison test. NAU: No Alcohol Use Group, AAU: Acute Alcohol Use Group, AUD: Alcohol Use Disorder Group

Table 4. Cox PH models to evaluate the main effect of alcohol use disorder and acute alcohol consumption on suicide reattempts

\begin{tabular}{|c|c|c|c|c|c|c|c|c|c|c|}
\hline & \multirow{2}{*}{ NAU } & \multicolumn{2}{|r|}{ AAU } & \multicolumn{2}{|r|}{ AUD } & \multirow{2}{*}{ AIC } & \multirow{2}{*}{ AAU } & \multicolumn{2}{|r|}{ AUD } & \multirow{2}{*}{ AIC } \\
\hline & & HR & $\mathrm{P}(\mathrm{CI})$ & HR & $\mathrm{P}(\mathrm{CI})$ & & & HR & $\mathrm{P}(\mathrm{CI})$ & \\
\hline Crude HR & Reference & 1.325 & $0.524(0.55-3.14)$ & 2.559 & $0.003(1.36-4.78)$ & 839.305 & Reference & 1.923 & $0.070(0.94-3.89)$ & 665.335 \\
\hline Model 1 & Reference & 1.449 & $0.470(0.53-3.96)$ & 3.175 & $0.004(1.45-6.91)$ & 550.514 & Reference & 2.432 & $0.041(1.03-5.69)$ & 426.611 \\
\hline Model 2 & Reference & 1.519 & $0.387(0.58-3.92)$ & 2.271 & $0.022(1.12-4.58)$ & 633.874 & Reference & 1.531 & $0.276(0.71-3.29)$ & 506.716 \\
\hline Model 3 & Reference & 2.190 & $0.160(0.73-6.54)$ & 2.962 & $0.015(1.23-7.10)$ & 447.576 & Reference & 1.784 & $0.211(0.72-4.42)$ & 352.851 \\
\hline
\end{tabular}

Model 1: Adjusted for demographic characteristics (age, sex, education level, marriage status, religion, employment, monthly income, smoking, and medical problems), Model 2: Adjusted for characteristics of suicide behaviors (suicide ideation, suicide plan, last will, past suicide attempt history, method of suicide attempt, medical lethality, and help seeking), Model 3: Adjusted for demographic and suicide behavior characteristics, CoxPH: Cox's proportional hazard, NAU: No Alcohol Use Group, AAU: Acute Alcohol Use Group, AUD: Alcohol Use Disorder Group, AIC: Akaike Information Criterion, HR: hazard ratio, CI: confidence interval 


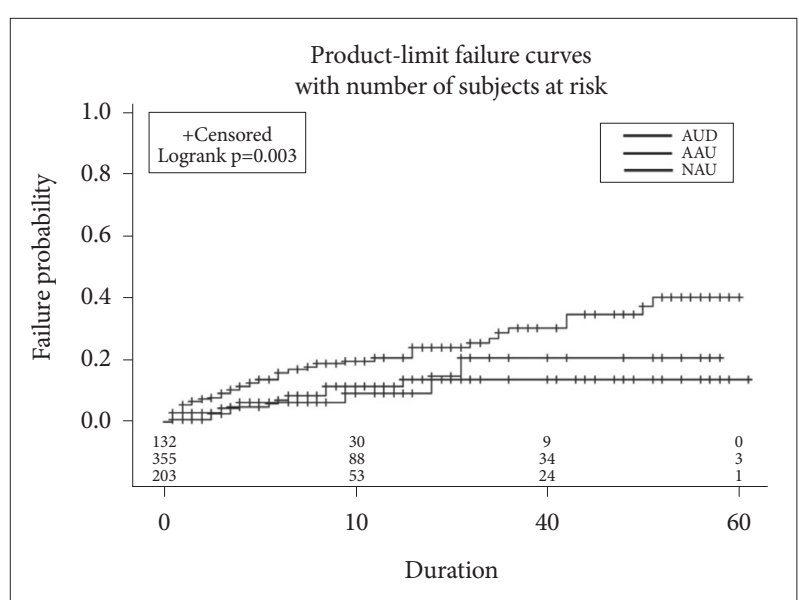

Figure 2. Kaplan-Meier estimates of cumulative incidence of suicide reattempt among study participants, stratified by type of alcohol use pattern. NAU: No Alcohol Use Group, AAU: Acute Alcohol Use Group, AUD: Alcohol Use Disorder Group.

$\mathrm{p}=0.001$ ). The survival analysis revealed that the risk of suicide reattempt was significantly higher in the AUD group than it was in the others, throughout the 60-month follow-up period. Further, as a result of drawing a hazard curve for each variable, it was confirmed that the proportional hazard exhibited valid intersections (Figure 2). Analysis of the difference in the reattempt period according to alcohol use patterns found that the increase in the cumulative proportion of suicide reattempts in patients with AUD lasted for a longer period than it did for the others ( 60 months vs. NAU: 36 months and AAU: 25 months; $\log \operatorname{Rank} \chi^{2}=11.34, \mathrm{p}=0.003$ ).

\section{DISCUSSION}

In the present study, we highlighted several important associations among characteristics of suicidal behaviors, risk of suicide reattempt, and alcohol use patterns among suicide attempters who visited emergency room. The present analysis revealed a high rate of incidence of AUD $(n=355,51.4 \%)$ in suicide attempters who visited the emergency room. This prominent association of alcohol with suicide behavior may be due to the cultural differences in alcohol drinking observed in Korea. The main findings of this study are as follows. First, we found that patients with AUD attempted suicide with more help seeking behaviors and had higher proportion of low medical lethality compared to not only NAU but also AAU group. Second, suicide attempters with AUD showed lower risk-rescue rating scores with a high rescue opportunity but suicide attempters with only AAU did not. Third, while AAU was not associated with the risk of future suicide reattempt before and after adjusting for other risk factors, AUD was related to higher risk of suicide reattempt compare to NAU and the increased risk was prolonged longer period.
This finding is consistent with those of previous studies that revealed that impulsive suicide attempts are more strongly associated with AUD than with AAU. ${ }^{18}$ The present study also replicated previous studies that AUD was found to increase the risk of repeated suicide attempts, ${ }^{19}$ and previous suicide attempts in alcohol dependent patients increased the risk of subsequent suicide attempts. ${ }^{20}$ While in line with prior work, the unique findings of this study are that the risk of suicide reattempts in patients with AUD was consistently high regardless of AAU, both before and after adjusting for other risk factors for repeated suicide attempt such as lethality and suicidal intent of the previous suicide attempt.

The results of this study reflect the fact that, among those with AUD, suicidal behavior is more related to the psychopathology of alcohol dependence, such as difficulty in impulse control. In the study, suicide attempts in patients with AUD tended to have low lethality and risk-rescue rating scores at baseline. Several previous studies have focused on this issue, and have reported that alcohol-related suicide attempts have low mortality and high risk of repeated suicide attempts. This tendency is called as "deliberate self-harm syndrome"..1 Therefore, suicide attempts in those with AUD may be overlooked due to their low lethality, as revealed in the present study (e.g., there is a lower risk of complete suicide in patients with AUD). However, based on the present findings, it is suggested that suicide attempts in patients with AUD require more careful evaluation and intervention owing to the high risks of repetition.

Furthermore, we also found that the high risk of suicide reattempt for patients with AUD lasted for more than 60 months after the previous suicide attempt, while the same persisted for up to 36 months in patients with AAU. This suggests that AUD may have more extensive and long-lasting impacts on subsequent suicidal behavior. It is important to note that some mood disorders, one of the important risk factor of suicide attempts, may be caused by alcohol consumption and alcohol withdrawal, and it is often associated with chronic affective dysregulation. ${ }^{22}$ Both aggression and depression are well-known risk factors for subsequent suicidal behavior in alcohol dependent individuals. ${ }^{23}$ As has been noted by numerous studies, AUD may play a key role in recurrent suicidal behavior, through a number of mechanisms that exacerbate negative mood states and risky behaviors that promote suicidal behaviors. ${ }^{24}$ On the other hand, some previous studies have explained the adverse consequences on brain development and executive function in patients with AUD due to a number of neurotransmitter hypofunctions. ${ }^{25}$ Thus, neurobiological factors, including serotonergic mechanisms, may play a role in repeated suicide attempts in patients with AUD. The serotonin neurotransmission deficiency may be associated with impulsiveness and suicide attempts that accompany alcohol depen- 
dence. ${ }^{26}$ It is well known that behaviors associated with prefrontal brain dysfunction in patients with AUD significantly predict not only the executive function system but also affective abnormalities. ${ }^{27}$ These permanent and complex effects of AUD in suicide attempters may increase and sustain the risk of repeated suicide attempts.

The strengths of this register-based study include the large numbers of cases of suicide attempters included in the analysis and the use of a detailed questionnaire to collect information on alcohol use pattern and characteristics of suicidal behaviors. Additionally, we performed follow-up and analysis of suicide reattempts after adjusting the detailed variables over a sufficient periods of time, which has never been done before in Korea. The present study has a number of limitations that should be considered when interpreting the findings. First, the sample of the present study was limited to one university hospital in a specific area in Korea, which may limit the generalizability of the current findings. Second, there was a selection bias because we excluded several patients who refused to consent to psychiatric evaluation or were missing drinking information. Third, there is insufficient information on comorbid diseases (e.g., mood disorder, personality disorder) that may affect the progress of suicide attempters. Despite these limitations, this study made it possible to understand the relationships among the factors related to alcohol use patterns that affect suicidal behavior over time.

In conclusion, suicide attempters with AUD have been shown to have lower lethality of suicide attempt but higher risk of suicide reattempts in the future, regardless of alcohol use at the time of suicide attempt. The results of present study suggest that suicide attempters with AUD should be considered a high-risk group for future suicide reattempts, and careful attention should be paid to the development of a longerterm intervention program. Additionally, each suicide attempter needs to be evaluated carefully for AUD, regardless of whether they had consumed alcohol at the time of the recent suicide attempt. Our results highlight the importance of specific and individualized aftercare programs of sufficient duration for suicide attempters with AUD. In this context, besides suicide prevention and aftercare programs that usually focus on depression, ${ }^{28}$ the careful assessment and appropriate treatment for underlying AUD among suicide attempters should be considered.

\section{REFERENCES}

1. Ezzati M, Lopez AD, Rodgers A, Vander Hoorn S, Murray CJ; Comparative Risk Assessment Collaborating Group. Selected major risk factors and global and regional burden of disease. Lancet 2002;360: 1347-1360.

2. Cavanagh JT, Carson AJ, Sharpe M, Lawrie SM. Psychological autopsy studies of suicide: a systematic review. Psychol Med 2003;33:395-405.
3. Koller G, Preuss UW, Bottlender M, Wenzel K, Soyka M. Impulsivity and aggression as predictors of suicide attempts in alcoholics. Eur Arch Psychiatry Clin Neurosci 2002;252:155-160.

4. Flensborg-Madsen T, Knop J, Mortensen EL, Becker U, Sher L, Grønbæk M. Alcohol use disorders increase the risk of completed suicideirrespective of other psychiatric disorders. A longitudinal cohort study. Psychiatry Res 2009;167:123-130.

5. Borges G, Rosovsky H. Suicide attempts and alcohol consumption in an emergency room sample. J Stud Alcohol 1996;57:543-548.

6. Boenisch S, Bramesfeld A, Mergl R, Havers I, Althaus D, Lehfeld H, et al. The role of alcohol use disorder and alcohol consumption in suicide attempts-A secondary analysis of 1921 suicide attempts. Eur Psychiatry 2010;25:414-420.

7. Lejoyeux M, Huet F, Claudon M, Fichelle A, Casalino E, Lequen V. Characteristics of suicide attempts preceded by alcohol consumption. Arch Suicide Res 2008;12:30-38.

8. Pirkola SP, Isometsa ET, Heikkinen ME, Lonnqvist JK. Suicides of alcohol misusers and non-misusers in a nationwide population. Alcohol Alcohol 2000;35:70-75.

9. Hawton K, Fagg J, McKeown SP. Alcoholism, alcohol and attempted suicide. Alcohol Alcohol 1989;24:3-9.

10. OECD. Suicide rates (indicator). Available at: doi: 10.1787/a82f3459-en. Accessed November 7, 2018.

11. Wojnar M, Ilgen MA, Jakubczyk A, Wnorowska A, Klimkiewicz A, Brower KJ. Impulsive suicide attempts predict post-treatment relapse in alcohol-dependent patients. Drug Alcohol Depend 2008;97:268-275.

12. Hillbrand M, Krystal JH, Sharpe KS, Foster HG. Clinical predictors of self-mutilation in hospitalized forensic patients. J Nerv Ment Dis 1994; 182:9-13.

13. Weisman AD, Worden JW. Risk-rescue rating in suicide assessment. Arch Gen Psychiatry 1972;26:553-560.

14. Misson H, Mathieu F, Jollant F, Yon L, Guillaume S, Parmentier C, et al. Factor analyses of the Suicidal Intent Scale (SIS) and the Risk-Rescue Rating Scale (RRRS): toward the identification of homogeneous subgroups of suicidal behaviors. J Affect Disord 2010;121:80-87.

15. Babor TF, Kranzler HR, Lauerman RJ. Early detection of harmful alcohol consumption: comparison of clinical, laboratory, and self-report screening procedures. Addict Behav 1989;14:139-157.

16. Bush K, Kivlahan DR, McDonell MB, Fihn SD, Bradley KA. The AUDIT alcohol consumption questions (AUDIT-C): an effective brief screening test for problem drinking. Arch Intern Med 1998;158:1789-1795.

17. Seong JH, Lee CH, Do HJ, Oh SW, Lym YL, Choi JK, et al. Performance of the AUDIT alcohol consumption questions (AUDIT-C) and AUDIT$\mathrm{K}$ question 3 alone in screening for problem drinking. Korean J Fam Med 2009;30:695-702.

18. Cherpitel CJ, Borges GLG, Wilcox HC. Acute alcohol use and suicidal behavior: a review of the literature. Alcohol Clin Exp Res 2004;28:18s28s.

19. Haukka J, Suominen K, Partonen T, Lonnqvist J. Determinants and outcomes of serious attempted suicide: a nationwide study in Finland, 19962003. Am J Epidemiol 2008;167:1155-1163.

20. Buri C, von Bonin B, Strik W, Moggi F. Predictors of attempted suicide among Swiss patients with alcohol-use disorders. J Stud Alcohol Drugs 2009;70:668-674.

21. Pennel L, Quesada JL, Begue L, Dematteis M. Is suicide under the influence of alcohol a deliberate self-harm syndrome? an autopsy study of lethality. J Affect Disord 2015;177:80-85.

22. Bagge CL, Sher KJ. Adolescent alcohol involvement and suicide attempts: toward the development of a conceptual framework. Clin Psychol Rev 2008;28:1283-1296.

23. Conner KR, Cox C, Duberstein PR, Tian L, Nisbet PA, Conwell Y. Violence, alcohol, and completed suicide: a case-control study. Am J Psychiatry 2001;158:1701-1705.

24. Zhang J, Wieczorek W, Conwell Y, Tu XM, Wu BY, Xiao S, et al. Characteristics of young rural Chinese suicides: a psychological autopsy study. 
Psychol Med 2010;40:581-589.

25. Harper C. The neuropathology of alcohol-specific brain damage, or does alcohol damage the brain? J Neuropathol Exp Neurol 1998;57:101-110.

26. Li D, He L. Meta-analysis supports association between serotonin transporter (5-HTT) and suicidal behavior. Mol Psychiatry 2007;12:47-54.
27. Gorwood P. Biological markers for suicidal behavior in alcohol dependence. Eur Psychiatry 2001;16:410-417.

28. Szanto K, Kalmar S, Hendin H, Rihmer Z, Mann JJ. A suicide prevention program in a region with a very high suicide rate. Arch Gen Psychiatry 2007;64:914-920. 